\title{
EL APROVECHAMIENTO TRADICIONAL DE LA DEHESA BOYAL EN UN ÁREA DE MONTAÑA DEL CENTRO DE ESPAÑA. PUEBLA DE LA SIERRA (MADRID)
}

\author{
POR \\ EVA MARTÍN JIMÉNEZ* \\ FERNANDO PARDO NAVARRO* \\ LUIS GIL SÁNCHEZ*
}

\section{Introducción}

La necesidad de los recursos del bosque y su conservación a largo plazo originaron la aparición de distintas formas de aprovechamiento, cuyos precedentes se sitúan, al menos en la Edad Antigua. Así por ejemplo en el Tratado General de Agricultura de Catón (siglo II a.C.) se mencionan dos tipos de aprovechamiento del bosque que tendrán gran importancia hasta tiempos recientes: silva caedua (monte bajo para la corta de leña) y silva glandaria (monte abierto o «hueco» para la obtención de fruto, generalmente bellota, para el ganado) (Meiggs, 1982). Estos dos tipos de aprovechamiento estaban en relación con el tamaño de los árboles que caracterizaban a cada uno de ellos, así como con la ecología de las especies que los componían. El monte bajo se aplicaba a árboles de pequeño tamaño y con buena capacidad de rebrote vegetativo, como robles o encinas, cuya corta estaba permitida por las leyes del momento, o suponía una pena menor que en el caso de los grandes árboles característicos de los montes huecos. Desde tiempos antiguos la corta de los árboles mayores estuvo castigada con penas mayores que la

* Eva Martín Jiménez; Fernando Pardo Navarro y Luis Gil Sánchez. Escuela Técnica Superior de Ingenieros de Montes. U.P.M.

Estudios Geográficos, LXIV, 252, 2003

$$
-407-
$$


de los pequeños. Así quedó reflejado por ejemplo en el Fuero Juzgo Visigodo (del siglo IV d.C.) en el que se establecía una pena de un sueldo por la corta de los árboles de pequeño tamaño, mientras que la pena por la corta de los árboles mayores era el doble (Scott, 1910).

Los montes bajos y los montes huecos se generalizaron durante la Edad Media. En España, esta época supone la implantación de la ganadería ovina trashumante como una de las principales formas de uso del territorio a lo largo de todo el país. Esto supuso que el bosque y el ganado entraran en competencia en no pocas ocasiones, apareciendo la dehesa, un tipo de espacio forestal característico de los paisajes españoles. La dehesa (del latín defensa, defendida, acotada) era un espacio forestal protegido de la entrada del ganado que se alimentaba en los pastos comunales abiertos (Montero et al., 1998). Una parte importante de las dehesas servían para la manutención y cobijo del ganado de labor (dehesas boyales), vital dentro de una economía de autoconsumo en la cual la fuente principal de carbohidratos procedía de la agricultura cerealista local, cuya fuerza de tiro principal eran los bueyes y las mulas (Mangas, 1981). Dada la necesidad de los recursos del bosque (leñas, maderas de construcción, frutos y hojas para el ganado) se establecieron sistemas, que pretendían ser renovables, mediante los cuales se pudiera asegurar la permanencia de los árboles en el futuro. De esta forma el hombre medieval aprovecha el bosque de manera intensa pero también conservadora (Rackham, 1990), lo que exigió una compleja reglamentación y la existencia de guardas especiales de los bosques (Torquemada, 1997), que evitaran la explotación abusiva, que era frecuente en momentos de falta de poder o bajo una presión demográfica intensa, en función del vecindario y la extensión del término municipal.

Los modelos de gestión del bosque de estas sociedades preindustriales se vieron sometidos a los avatares de la historia. El uso comunal de muchas dehesas pasó a ser un uso exclusivo de los ayuntamientos cuando éstas se convirtieron en terrenos de propios (García de Cortazar, 1990). A raíz de este cambio los consistorios trataron de sacar beneficio de las dehesas arrendando sus pastos y su bellota o vendiendo su leña. El cambio de la primitiva función comunal de la dehesa dio lugar a una explotación abusiva y a la degradación final del espacio silvopastoral. Sin embargo donde se han mantenido los modelos de gestión de la dehesa originales o próximos al modelo primitivo, estos pueden ser de utilidad a la hora de buscar fórmulas que permitan 
compatibilizar el uso tradicional del bosque con la conservación de sus valores naturales.

En el presente trabajo se analiza la gestión tradicional de una dehesa boyal localizada en el Sistema Central español, en el municipio madrileño de Puebla de la Sierra (Madrid). El objetivo principal ha sido conocer las características de la gestión tradicional y sus efectos sobre el bosque. Además se analiza la compatibilidad del uso tradicional de la dehesa con la gestión de este espacio natural con vistas a su conservación. Se estudian las prácticas silvopastorales que llegaron hasta el siglo xx por transmisión oral, cuyo conocimiento está en la memoria de las personas de mayor edad. Se han utilizado dos fuentes de información. Una de ellas de carácter general, procedente de distintos documentos escritos referidos al área de estudio. La otra fuente de información ha sido obtenida de entrevistas guiadas a hombres y mujeres de edad comprendida entre 70 y 85 años. Estas han permitido reconstruir los usos y gestión de la dehesa, así como elaborar un mapa de la toponimia del lugar. Como información adicional se han utilizado los datos de un Inventario Forestal de la dehesa realizado recientemente (Martín, 2001).

\section{Área de estudio}

El área de estudio se encuentra en el tramo oriental del Sistema Central, entre Somosierra y las estribaciones más occidentales del Macizo de Ayllón (Fig. 1). Forma parte de la Sierra Norte de Madrid, comarca montañosa cuya población más importante es Buitrago de Lozoya. Este municipio fue durante el Antiguo Régimen la Villa del Señorío del mismo nombre (Grupo73, 1973). Puebla de la Sierra se sitúa en el límite oriental del antiguo Señorío de Buitrago. Su término municipal, que consta de 5757 ha, aparece configurado por un valle de montaña limitado al este y al oeste por sendas alineaciones montañosas (Fig. 2). El núcleo de población se sitúa a $1161 \mathrm{~m}$, siendo las cotas máximas el pico de La Tornera (1866 m) y la Peña de la Cabra (1834 m). Desde el siglo Xvı la población ha oscilado entre los 250 y los 350 habitantes con escasas variaciones hasta los años 70 del siglo $\mathrm{xx}^{1}$ (Fernández, 1980). Esto supone

\footnotetext{
${ }^{1}$ Los datos de población han sido consultados en Fernández (1980) (siglos XVI y XVII), Catastro de Ensenada (siglo XVIII), Diccionario de Madoz (siglo XIX) y censos de población del Instituto Nacional de Estadística (siglo Xx).
} 


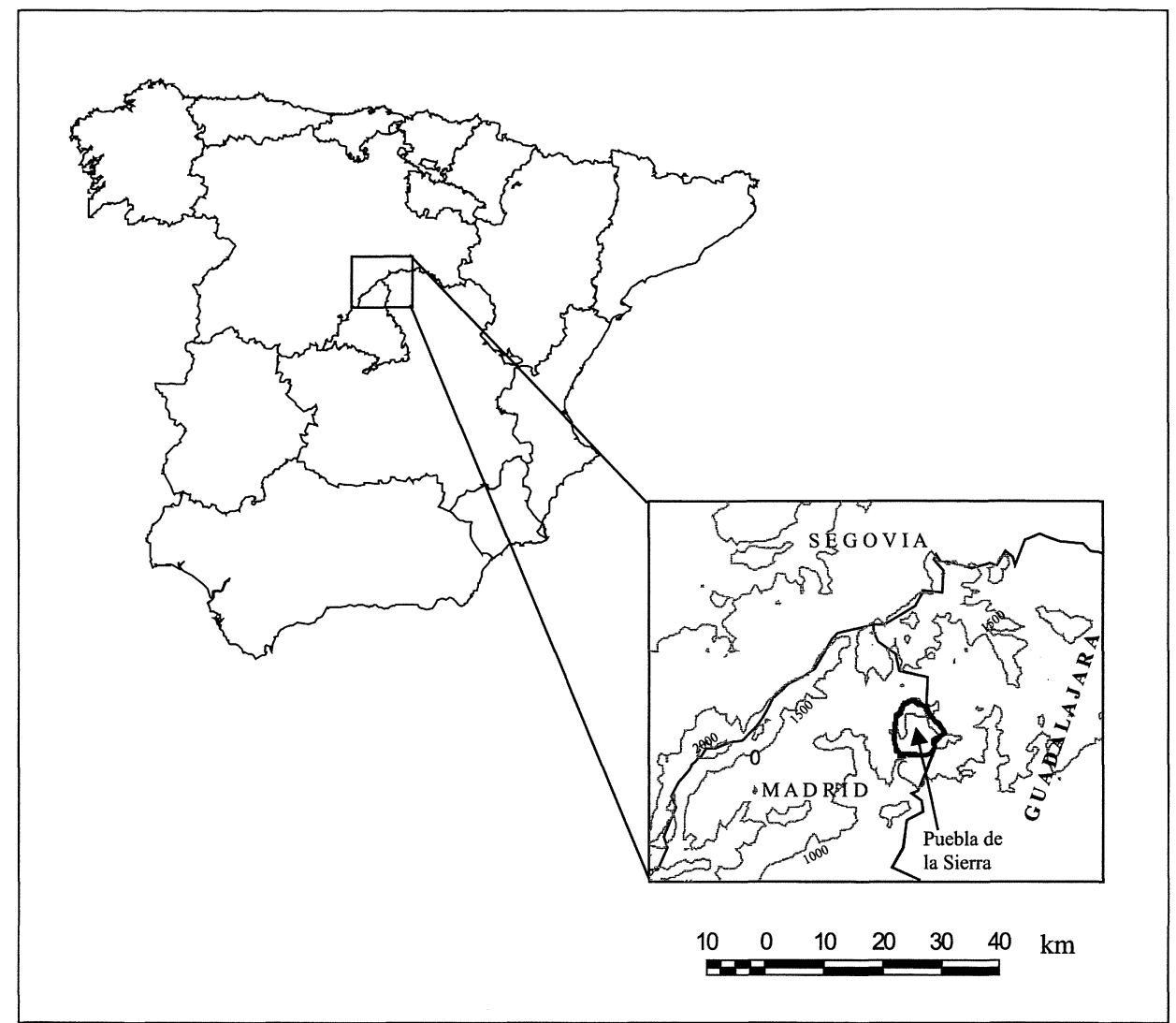

Fig. 1.-Localización del área de estudio.

una bajísima densidad de población (entre 4 y 6 habitantes $/ \mathrm{km}^{2}$ ) para el amplio territorio que ocupa su término municipal. A partir de 1960, ya en el siglo xx el descenso es más acentuado situándose en 1991 con 48 habitantes. En la actualidad el número de habitantes censados (censo de 2001) es de 96, aunque la población permanente en el municipio es de 44 vecinos.

La litología predominante del término municipal está compuesta por rocas metamórficas (cuarcitas y pizarras) procedentes de sedimentos ordovícicos y silúricos. Sobre estas rocas se originan suelos con texturas de groseras a medias, cuya erosión se ha visto favorecida por la deforestación y la elevada pendiente. El clima se caracteriza por la

Estudios Geográficos, LXIV, 252, 2003 


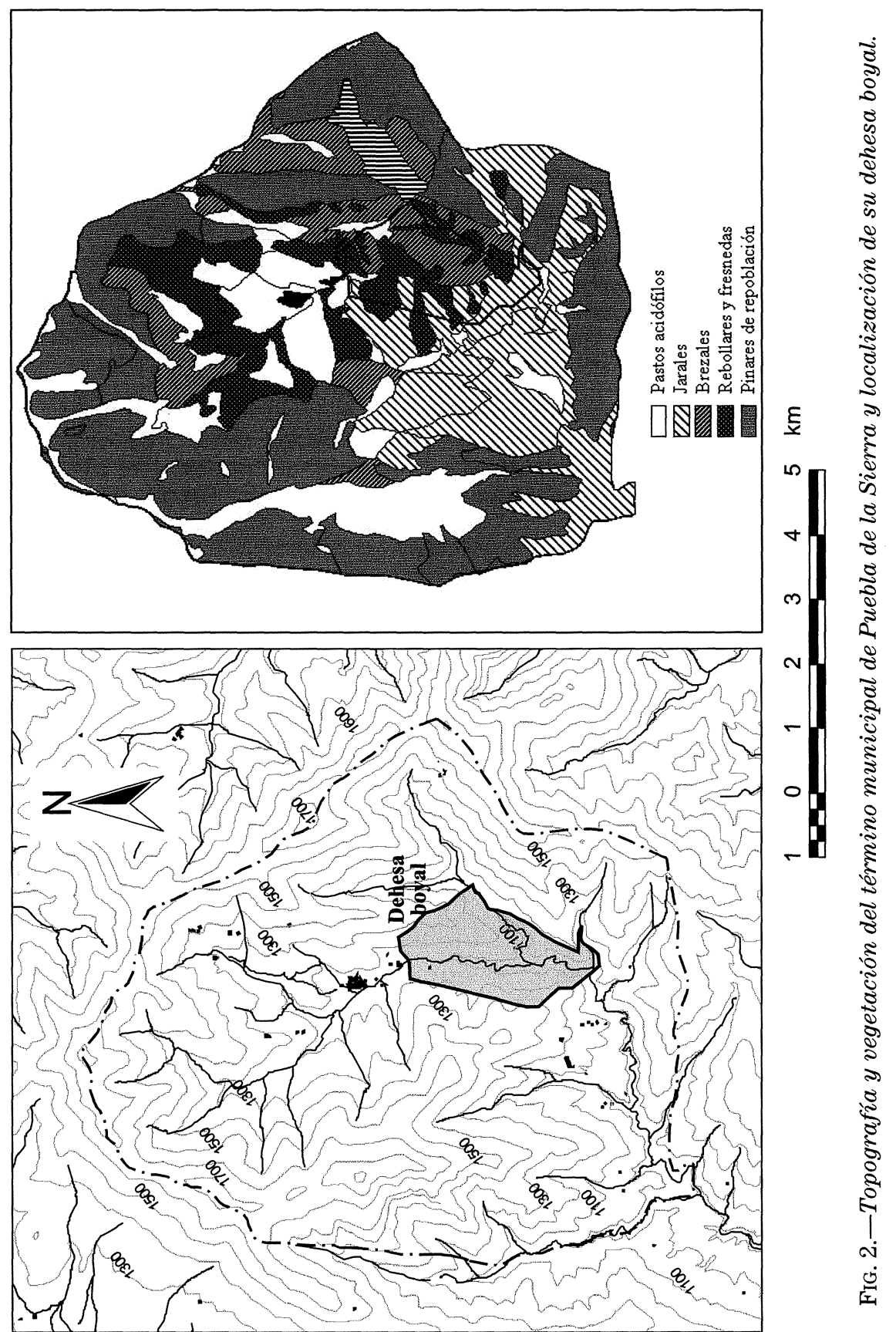

Estudios Geográficos, LXIV, 252, 2003 
mediterraneidad, la continentalidad y los efectos de la orografía, teniendo una precipitación anual inferior a los 900 milímetros, un prolongado periodo de heladas y una acusada sequía estival.

La Dehesa Boyal de La Puebla, con 336 hectáreas ocupa el 5,8 por ciento de la superficie del término y se localiza al sur del núcleo de población (Fig. 2). Tiene una posición estratégica dentro del término en cuanto a su proximidad al casco urbano y su localización intermedia respecto a los pastos localizados a mayor altitud. Posee una forma alargada con orientación general norte-sur y está atravesada, en esta dirección, por el arroyo de la Puebla. Este divide a la dehesa en dos mitades quedando configurada por dos laderas de elevada pendiente que convergen en el citado arroyo. En su cuadrante sureste se encuentra el arroyo del Portillo. Los otros pequeños cursos de agua, que de forma permanente la atraviesan, y las diferentes exposiciones de sus laderas, lomas y vaguadas dotan a la dehesa de una gran diversidad. Casi las dos terceras partes están ocupadas por jarales y brezales, existiendo una superficie de melojar (Quercus pyrenaica) cercana al 40 por ciento.

\section{La gestión tradicional de la Dehesa de la Puebla}

Antecedentes históricos. - Las normas más antiguas sobre la explotación de los bosques de la comarca que conocemos aparecen en el Fuero de Sepúlveda, Comunidad de Villa y Tierra a la que perteneció toda la vertiente sur del Macizo de Ayllón (incluida la Sierra de la Puebla) tras la reconquista del Reino Árabe de Toledo en 1085(Sáez, 1953). Existen numerosos títulos del Fuero que se refieren al aprovechamiento de los bosques. En el denominado «de cómo se deben de esquilmar los árboles», se establece que el roble no se podía «esquilmar» en el «tiempo de la lande ${ }^{2}$ (bellota)». Fuera de este periodo solo se podían cortar «de medio arriba, mas nil' eche (derribe o tale) nil' descogolle (descabece)».

En 1256 el Concejo de Buitrago se segrega del de Sepúlveda tras la concesión del Fuero Real por Alfonso X (Fernández, 1980). El Fuero hace referencia al uso comunal de dehesas y montes: «que sean libres $e$ quitas... ... e lo que dent saliere que lo metan en pro del concejo». En

${ }^{2}$ Del latín glans, glandis, bellota

Estudios Geográficos, LXIV, 252, 2003

$$
-412-
$$


el año 1366 la Comunidad de Buitrago es cedida por Enrique de Trastámara a Pedro González de Mendoza. Se establece un Régimen Señorial en el cual el aprovechamiento de los montes quedaba regulado por las Ordenanzas de Villa y Tierra, cuyas copias más antiguas conservadas corresponden al siglo XVI (Fernández, 1966; Flaquer, 1979). Para vigilar su cumplimiento existían guardas de los montes. En el preámbulo de algunas Ordenanzas de Villa y Tierra se recalcaba expresamente la vocación ganadera de la comarca. Así en las de 1573 se decía: «... lo principal que al bien común de la dha villa y tierra conviene es la cría $y$ conservación de los montes porque la tierra es más conviniente para ganados que para panes y viñas...». Estas mismas Ordenanzas expresaban una preocupación por las talas e incendios realizados en tiempos anteriores, hechos que mermaban el aprovechamiento ganadero de los montes comunes. Por este motivo se trataron de endurecer las normas que protegían los montes en algunos de sus aspectos ${ }^{3}$.

Las Ordenanzas especificaban el número de árboles que se debía dejar cuando se rozaran terrenos para su cultivo. Se establecía que si la densidad de matas (de roble) era muy alta, algunas podían ser arrancadas, siempre que se dejara un árbol en cada cuadrado de 20 por 20 varas $^{4}$. Esta norma se refería a terrenos que no fueran comunes de Villa y Tierra ya que en éstos el cultivo estaba prohibido, de forma que el ganado pudiera aprovechar durante todo el año sus pastos y bellota. Las Ordenanzas de 1583 declaraban que el roble podía ser cortado siempre que fuera de pequeño tamaño (de más de «marco») ${ }^{5}$. La protección de los robles mayores (de más de «marco») era aplicable a cualquier tipo de terreno, incluidos los de pro-

\footnotetext{
${ }^{3}$ En el preámbulo de las Ordenanzas de 1573 se decía lo siguiente: «...que por experiencia se ha visto que para criar y conservar los dichos montes demás de las ordenanzas que están hechas conviene añadir algunas otras, en especial que no se pudiere rozar ni sacar de quaxo en los montes y términos comunes... ... y que ningún roble ni de más de marco ni de menos de marco no se pudiese cortar sin licencia de la justicia... ... y que para leña solo se pudiese cortar lo seco por pie y rama y lo verde por rama, dexando alero y pendolero, y que en los tercios tampoco se pueda sacar de quaxo o a lo menos que en cada mata se dexe un rrobre o dos con sus raíces... ... y ansí mismo se podrían rrayar algunos pedazos de tierras de lo común e irlos bedando de diez en diez años para que allí sepuediese dar licencia para cortar y otras cosas»

${ }^{4}$ La vara era una unidad de medida lineal que, en Castilla, equivalía a $0,836 \mathrm{~m}$. Esto suponía, que para un cuadrado de 20 por 20 varas, se dejaba un árbol por cada $279,6 \mathrm{~m}^{2}$, es decir 36 árboles por ha.

${ }^{5}$ El tamaño se establecía en función del «marco», aunque en ninguno de los capítulos de las Ordenanzas se especifica la equivalencia del «marco» con ningún tipo de medida de grosor del fuste o de otras dimensiones del árbol.
} 
piedad privada. No obstante cada ayuntamiento podía cortar hasta 10 robles al año de sus dehesas, bajo licencia de la justicia de Buitrago, que se empleaban en la construcción de casas o edificios públicos. También se daban licencias para la corta de madera para hacer carros, cercas o arados. Aunque se permitía cortar ramas de los robles mayores, estos no podían ser desmochados y se obligaba dejar «dos horcas y un pendón» o «dos aleros y un pendolero ${ }^{6}$, debiéndose de hacer las podas desde el día de todos los santos hasta mediados del mes de abril. Esta norma era complementaria a la que prohibía cortar o podar robles que tuvieran bellota. En este caso se ordenaba que se amojonasen y vedasen los montes para que los ganados no entraran hasta que se diera la licencia correspondiente.

Además de los datos generales sobre el uso de los montes aportados por las Ordenanzas de Buitrago se dispone de información más concreta sobre los montes de La Puebla al final del Antiguo Régimen a través del Catastro de Ensenada, que data de $1754^{7}$. En esta fecha la dehesa estaba ocupada por «pasto boyal» $\mathrm{y}$ «monte alto». Sus leñas y pastos eran para «las urgencias del común (de los vecinos)». La mayor parte del término municipal estaba ocupado por montes comunes de Villa y Tierra, con más del 85 por ciento de la superficie total de Puebla. Estos montes estaban compuestos de «peñascales, sierras, tomillos, jaras $y$ demás inútil» $\mathrm{y}$ «pasto de dehesa» principalmente; también el descrito como «monte alto para cortes de leña y maderas» que suponía únicamente el 5,5 del término, y lo constituían principalmente robles dispuestos «regularmente entre los pastos». Su corte estaba prohibido «no siendo de ramas dejando horca y pendón según reales estatutos». Algunos vecinos se dedicaban parte del año a la fabricación de carbón de brezo y de horquillas y palas de roble $^{8}$. Cabe pensar que la esti-

${ }^{6}$ La horca o el alero es una rama horizontal de la cual se colgaba a los condenados a pena de muerte. El pendón es un vástago que sale del tronco principal del árbol. La expresión dejar «horca y pendón» se convirtió en una forma general de señalar que debían dejar, por lo menos, una rama gruesa de la cual saliera un brote y no desmochar el árbol. Algunas Ordenanzas locales como las de Buitrago o el Sexmo segoviano de Lozoya iban más allá obligando a dejar dos y tres aleros respectivamente.

$7 \mathrm{El}$ Catastro de Ensenada ha sido consultado en la obra: «Trascripciones literales de las respuestas generales del "Catastro de Ensenada» correspondientes a los términos comprendidos en la actual provincia de Madrid» (trabajo mecanografiado no publicado) en la Biblioteca de la Consejería de Obras Públicas, Transportes y Urbanismo de la Comunidad de Madrid.

${ }^{8}$ Cabe pensar que la estimación del monte alto, hecha seguramente «a ojo», se referiría a un monte bastante abierto, hecho que explicaría una cifra tan baja. En la actualidad el monte alto supone más de la mitad de la superficie del término. 
mación del monte alto, hecha seguramente «a ojo», se referiría a un monte bastante abierto, hecho que explicaría una cifra tan baja.

La utilización de la dehesa en el siglo XX.-Tras la finalización del Antiguo Régimen y la pérdida de vigor de las Ordenanzas señoriales, los aprovechamientos de la dehesa pasaron a ser regulados por La Hermandad de Labradores y Ganaderos. Se trataba de una asociación de tipo formal y de carácter institucional, regida por el presidente, que mantuvo los usos y costumbres hasta la extinción de las actividades tradicionales a finales de 1970. El presidente organizaba los trabajos y suertes, estableciendo las tierras y periodos de guardar la Dehesa. Imponía además sanciones y multas, a través del alguacil y el guarda ${ }^{9}$, a aquellos vecinos que no cumpliesen las normas.

Los aprovechamientos de la dehesa se organizaban estacionalmente distribuyéndose por distintas zonas delimitadas por sendas y caminos (Fig. 3). Los caminos de herradura (de circunvalación e interiores) tenían un ancho medio de dos metros. Además existían veredas permanentes del ganado. La dehesa estaba dividida en tres zonas. En la parte central, en torno al valle principal, se encontraban «los huecos», delimitados por el camino de Torrelaguna al este y por el camino bajo a Robledillo al oeste. Ocupaban el 23 por ciento de las 336 hectáreas de la dehesa. El resto de la superficie, ascendiendo ladera arriba, eran «las orillas»(el 67 por ciento). La parte más externa de «las orillas» se denominaban «medias orillas», las cuales quedaban delimitadas por la vereda del Qüitocón al este y la vereda de los Castillejos al oeste y ocupaban el 36 por ciento de la superficie. En la parte sur de la dehesa se encontraba La Mueda, que era una zona de libre pastoreo, debido probablemente a lo abrupto del terreno, que ocupaba el 8 por ciento de la superficie de la dehesa.

$\mathrm{El}$ arreglo y mantenimiento de los caminos se organizaba entre los vecinos del pueblo por «adras» ${ }^{10}$, centrándose en tres días al año: el 13 de junio, San Antonio; el 25 de abril, San Marcos; y el 30 de noviembre, San Andrés. Los restos que hoy día se encuentran de los antiguos caminos, muretes de piedra, pasos de agua, empedrados, etc., muestran el ex-

\footnotetext{
${ }^{9}$ La figura de guarda mantuvo sus privilegios hasta casi nuestros días pudiendo cultivar de forma exclusiva tierras de vega destinadas a huerto, localizadas dentro de la dehesa, que actualmente llevan su nombre («Huerta del Guarda»).

${ }_{10}$ Brigadas formadas por unidades familiares que correspondían al mismo barrio dentro del pueblo.
} 


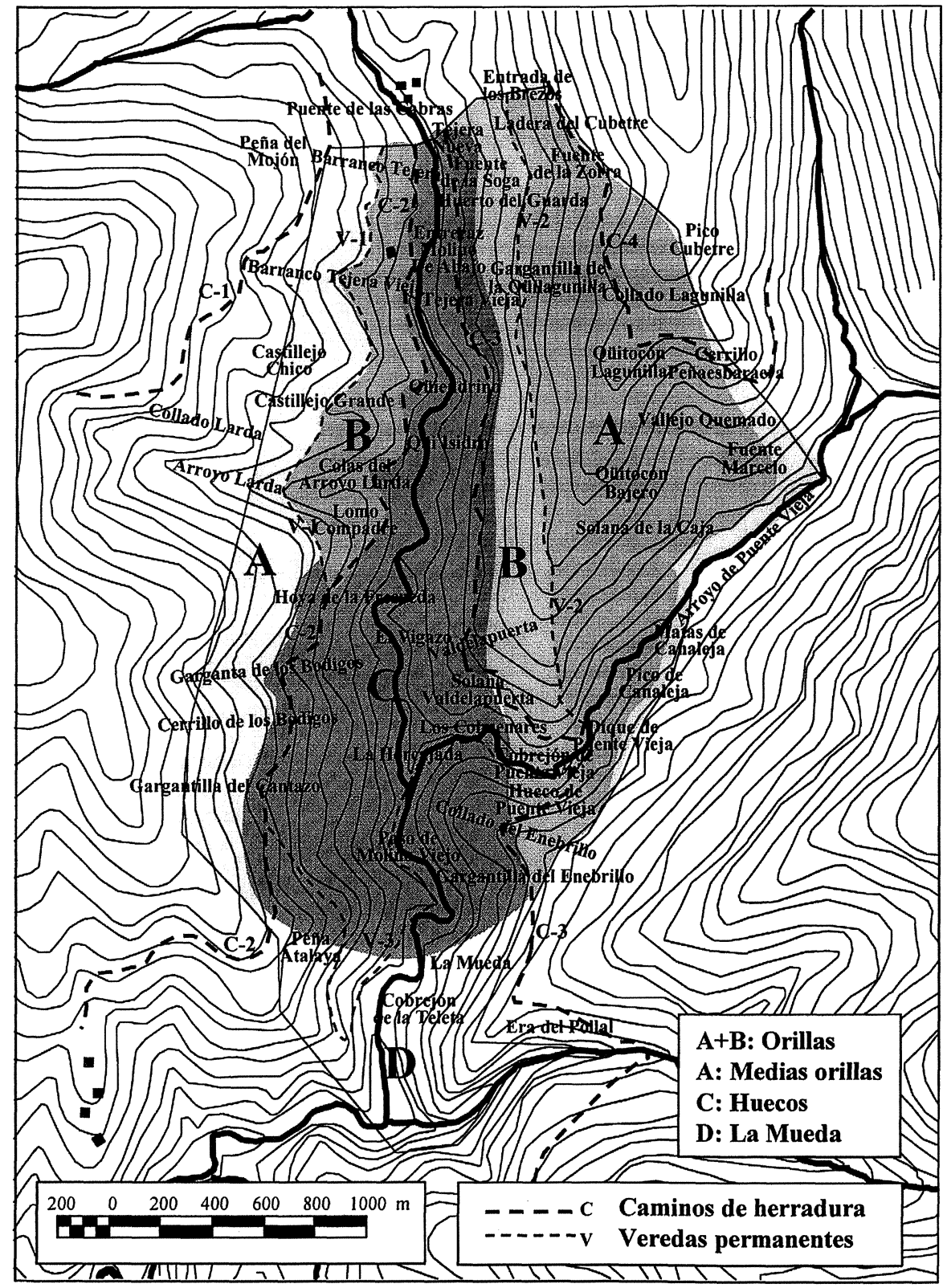

Fig. 3.-Mapa de topónimos, caminos y veredas de la dehesa. Se indica también la división de la dehesa en cuatro zonas, las cuales eran aprovechadas

tradicionalmente según un calendario estacional como se explica en el texto.

Estudios Geográficos, LXIV, 252, 2003 
cepcional trabajo comunal de todo un pueblo, mantenido por generaciones. Los topónimos que se han conservado a lo largo y ancho de toda la dehesa también son prueba del intenso y prolongado uso al que ha sido sometida (Fig. 3). Estos topónimos no solo hacen referencia a elementos naturales (Entrada de los Brezos, Qüiendrino u Hoya de la Fresneda) sinó también a elementos de origen humano (Tejera Nueva, Entrecaz, Molino de Abajo o Puente Vieja) y al aprovechamiento del bosque de la dehesa (Qüitocón, Vallejo Quemado o Matas de Canaleja).

Ganadería y agricultura.-El ganado de tiro podía pastar durante todo el año en la dehesa, aunque desde noviembre hasta San Marcos, 25 de abril, se solían guardar en el pueblo. Cuando se dejaban dentro se cerraban en el Corral de los Bueyes, de uso comunal, cerca de Valdelapuerta (Fig. 3). Las vacas y bueyes de todos los vecinos eran cuidados por el vaquero, al que se le pagaba en función del número de cabezas que poseía cada vecino. Además de los animales de tiro algunos vecinos también criaban anualmente un ternero que solía ser vendido. El ganado vacuno aprovechaba principalmente los huecos, ascendiendo a las partes altas de la Dehesa (orillas y medias orillas) en verano, donde tenían sus sesteaderos. La pared de piedra que rodeaba la dehesa impedía que pudiesen salir y ocasionar daños en el cultivo de cereal.

De noviembre a abril la dehesa se abría al resto del ganado, fundamentalmente ovejas y cabras (salvo La Mueda, de libre pastoreo). Este periodo, coincidente con las épocas en las que escaseaba la hierba y los rebaños se guardaban en el pueblo, se aprovechaba para la unión de los rebaños de hembras con los machos y para el saneamiento de los ganados. Este último consistía en el sacrificio de las cabras y ovejas viejas al mismo tiempo que se hacía la matanza de los cerdos. Se seguían así unas prácticas y rutinas pastorales que agrupaban los partos, en el caso del ovino a principios de la primavera y en el caprino en verano.

Desde primeros de abril hasta el quince de julio se abrían las «medias orillas». A mediados de septiembre, antes de la caída de la hoja, se abrían las «orillas», para el aprovechamiento de la «barda» (hojas de roble), «fresniza» (hoja de fresno), y leña de robles y fresnos. El forraje se guardaba en las «casillas» del pueblo para alimentar al ganado en el invierno. $\mathrm{El}$ «sacer» (Acer monspesulanum) se «parrotaba», tirándose las hojas sobre el terreno para que el ganado las comiese en verde. Para los Santos, día 1 de noviembre, se «derrotaba» la Dehesa. Esto suponía 
que se abrían «los huecos» (los mejores pastos de la dehesa), como La Fuente de la Soga, Valdelapuerta, Los Colmenares, La Horcajá, etc., permitiéndose a los vecinos el pastoreo salvo en aquellos lugares vedados (zonas de «tallar» destinadas al aprovechamiento en monte bajo).

En la Dehesa también se recolectaban la bellota, que posteriormente se almacenaba para la alimentación del ganado en invierno. Los vecinos interesados realizaban el trabajo en grupo y al final de cada jornada se reunían en el Ayuntamiento para repartir de forma equitativa el total de lo recogido con el fin de compensar a aquellos que les hubiese tocado zonas peores, de menor producción o más abruptas. El reparto era por medias (dos medias equivalen a una fanega, es decir 55,5 litros).

Como complemento de la actividad ganadera se practicaba la roza, quema y siembra de algunas zonas. Su periodicidad venía marcada por la expansión de jaras. Estas zonas eran abiertas, con orientaciones más de solana que de umbría y con una densidad escasa de robles ${ }^{11}$. La organización de los trabajos era de forma rotativa con un sistema de «adras». La roza se hacía en invierno y era manual, formándose montones con el matorral cortado. En septiembre, cuando los montones ya estaban secos, se procedía a su quema. Posteriormente se dispersaban las cenizas cuando se levantaba el terreno con el fin de preparar la siembra, que sólo se realizaba ese mismo año, con las primeras lluvias de octubre. Para ésta se concedían suertes, sembrándose trigo en las mejores tierras y centeno en las peores.

Aprovechamiento de madera y leñas. - La mayor parte de la dehesa esta formada por robles, en su mayoría Quercus pyrenaica, aunque también hay algunos de Quercus petraea. En general en toda la comarca se le da el nombre de roble, indistintamente, a ambas especies. Se suele hacer una distinción entre tipos de roble que no tiene en cuenta la especie pero sí la morfología del árbol. Cuando éste forma matas de las que salen varios vástagos, caso casi exclusivo de Quercus pyrenaica dada su gran capacidad de rebrote vegetativo, se le denomina «rebollo». Por el contrario cuando existe un árbol individualizado, con un solo vástago, se le da el nombre de «roble».

${ }^{11}$ Actualmente el jaral ocupa el $26,3 \%$ de la superficie de la dehesa, lo que supone 88,7 hectáreas. En el pasado su superficie debió ser notablemente menor por el control del matorral que haría el ganado, mucho más abundante que en la actualidad.

Estudios Geográficos, LXIV, 252, 2003 
En una zona en la que el invierno era especialmente duro la leña era de gran importancia para todos los vecinos. La leña era obtenida mediante la corta como monte bajo y por podas de los robles mayores, siendo éste último el tipo de aprovechamiento más frecuente. Los turnos de corta o poda oscilaban entre doce y catorce años. En las zonas dedicadas a monte bajo el terreno se acotaba durante 5 años al ganado ovino y 7 años al caprino.

La formación de los robles comenzaba cuando algún vecino optaba por respetar alguno de los robles menores que caían en sus suertes (Figs. 4-A y 4-B). También podía respetar algún «rebollón», es decir uno de los rebrotes de mayor tamaño en una mata de rebollos. Los robles seleccionados solían tener un diámetro de por lo menos 10 centímetros. En este momento se procedía a la poda de ramas laterales hasta la cabeza de un hombre. Una vez alcanzados los $30 \mathrm{~cm}$ de diámetro se le cortaba la guía principal, a una altura que dependía de los medios y pericia de cada vecino, empezando a considerarse un «roble joven». A partir de entonces los robles se «desmoñaban». Esta operación consistía en dejar varias ramas horizontales, o ligeramente inclinadas, de corta longitud que salían del fuste del árbol y cuyos brotes eran cortados cuando llegaba el turno (Fig. 4-C). De estas ramas, denominadas «pezones» 0 «pezoneras», surgían numerosos rebrotes, «renovizos», de los cuales se dejaba uno, ni muy fino ni muy grueso, de orientación preferentemente vertical, con lo que se favorecía la nueva brotación. En la siguiente poda se cortaba la rama reservada en la poda anterior en cada «pezón», para que ésta no cogiese demasiado «vicio», es decir para que no adquiriera un diámetro demasiado grande, estableciéndose un límite en torno a los $10 \mathrm{~cm}$. Cuando la rama reservada superaba este diámetro se dificultaba el desarrollo de nuevos brotes, impidiendo los futuros aprovechamientos de leñas del árbol. Una vez realizada la poda se volvía a guardar otra rama vertical. Con el tiempo los robles adquirían formas variadas, desde árboles con una sola cruz con varios pezones, a árboles con los pezones repartidos en varios pisos (Figs. 5-A y 5-B). En general se tendía a formar una copa con ramas horizontales compensadas, dándole al árbol un reparto equilibrado de las cargas.

Las leñas gruesas obtenidas de cortas o podas se destinaban principalmente a la producción de carbón, y las finas como combustible para los hogares. Además del carbón de roble también se producía carbón de brezo, muy apreciado para las fraguas por su alto poder calorífico. El 


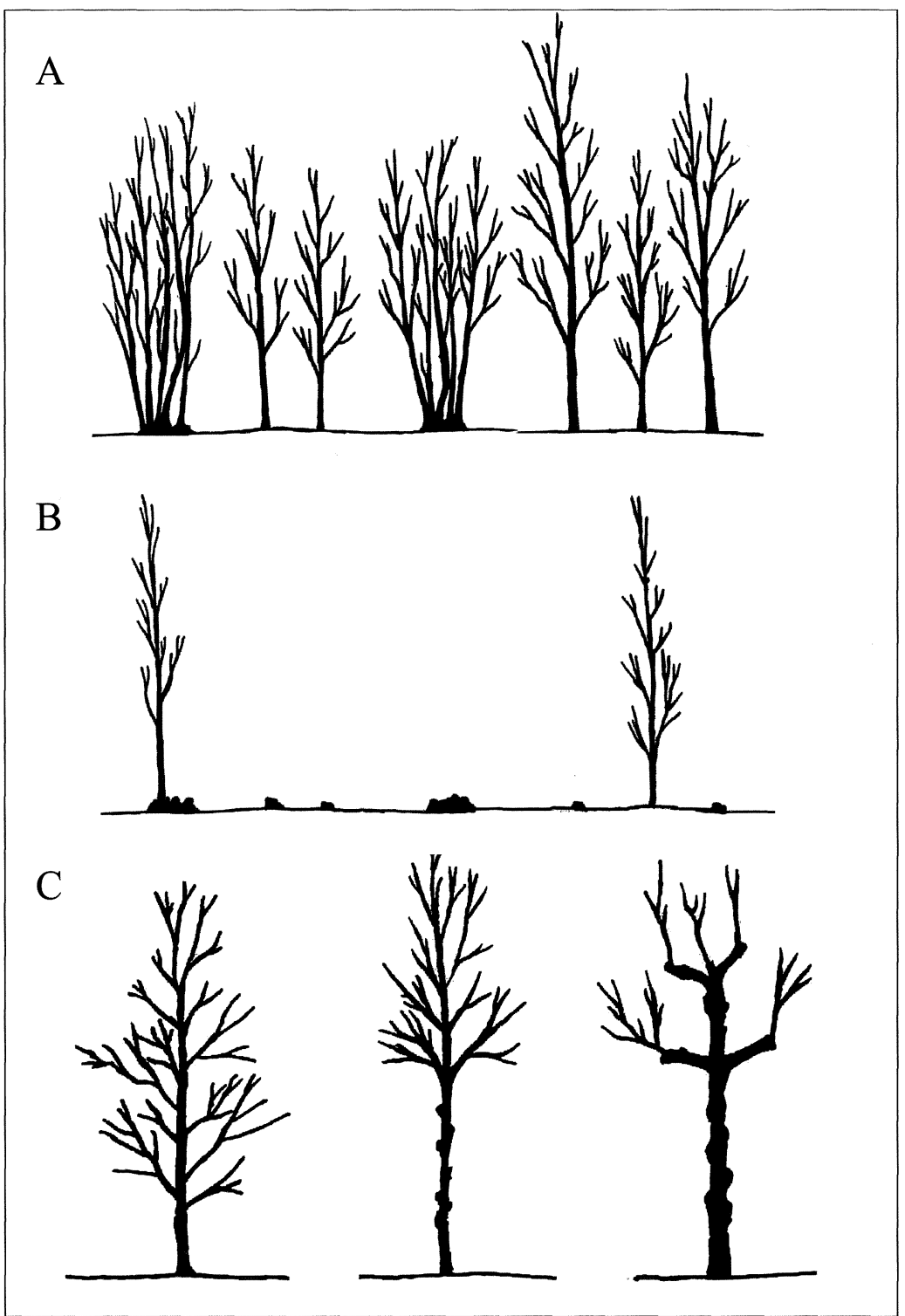

Fig. 4.-Primeras etapas de «formación de los robles» mediante podas. En A se representa un rodal de robles en el que hay, tanto matas como árboles de semilla. En $B$ se representa al mismo rodal después de haber cortado todos los árboles menos dos, uno de ellos correspondiente a uno de los vástagos de una mata («rebollón»). En C se representa, de izquierda a derecha, la evolución de la copa del árbol tras la realización de distintas fases de poda (ver texto).

Estudios Geográficos, LXIV, 252, 2003 
carbón se intercambiaba o vendía para el abastecimiento de otros productos, transportándolo en mulas hasta municipios del sur como Uceda, Alpedrete o Torrelaguna. La madera de los robles que se morían también era aprovechada para la fabricación de muebles, siempre que no presentara pudriciones. En ocasiones se apeaba algún roble de mayores dimensiones para su utilización como elemento estructural de casas, tinados y casillos. La madera de roble se utilizaba también en la fabricación de horquillos, mazas y astiles.

El sistema de reparto de leñas obtenidas de las podas o las cortas se hacía a través de suertes vecinales. Cada vecino tenía que pagar un canon anual por cada suerte, existiendo la posibilidad de que alguno de ellos pudiera disfrutar de más de una suerte. Esto lo hacían sobre todo aquellas familias cuya economía estuviese principalmente sustentada por el carbón. En una suerte entraban alrededor de ocho «robles» para trasmochar. Esto equivalía a unas setenta «seras» ${ }^{12}$, lo que suponía unos 8.000 a 10.000 kilos en verde, dando una media por árbol podado de unos 1.000 kilos. Para una población media de 300 habitantes, que fue la que se mantuvo hasta mitad del siglo xx, y una relación de 4 habitantes por vecino (Grupo 73, 1973), esto suponía la poda de $75 \times 8=600$ robles.

Los alisos (Alnus glutinosa) se aprovechaban igual que los robles. Su madera era muy apreciada para la construcción de comederos para el ganado. Los fresnos (Fraxinus angustifolia) y los sacer (Acer monspessulanum) se «escabechaban» (desmochaban), operación que consistía en cortar su guía principal a unos 2 metros de altura, para después cortar los brotes surgidos en esa zona cada ocho años (Fig. 5-C). El reparto de la fresniza y de su leña y varas hacia igualmente por suertes correspondiendo aproximadamente cuatro árboles a cada unidad familiar. Como en el caso de las suertes de leña cada vecino debía de pagar un canon anual por la suerte de fresniza.

\footnotetext{
${ }^{12}$ Dos seras son una carga de leña de un serón de mula o burro.
} 


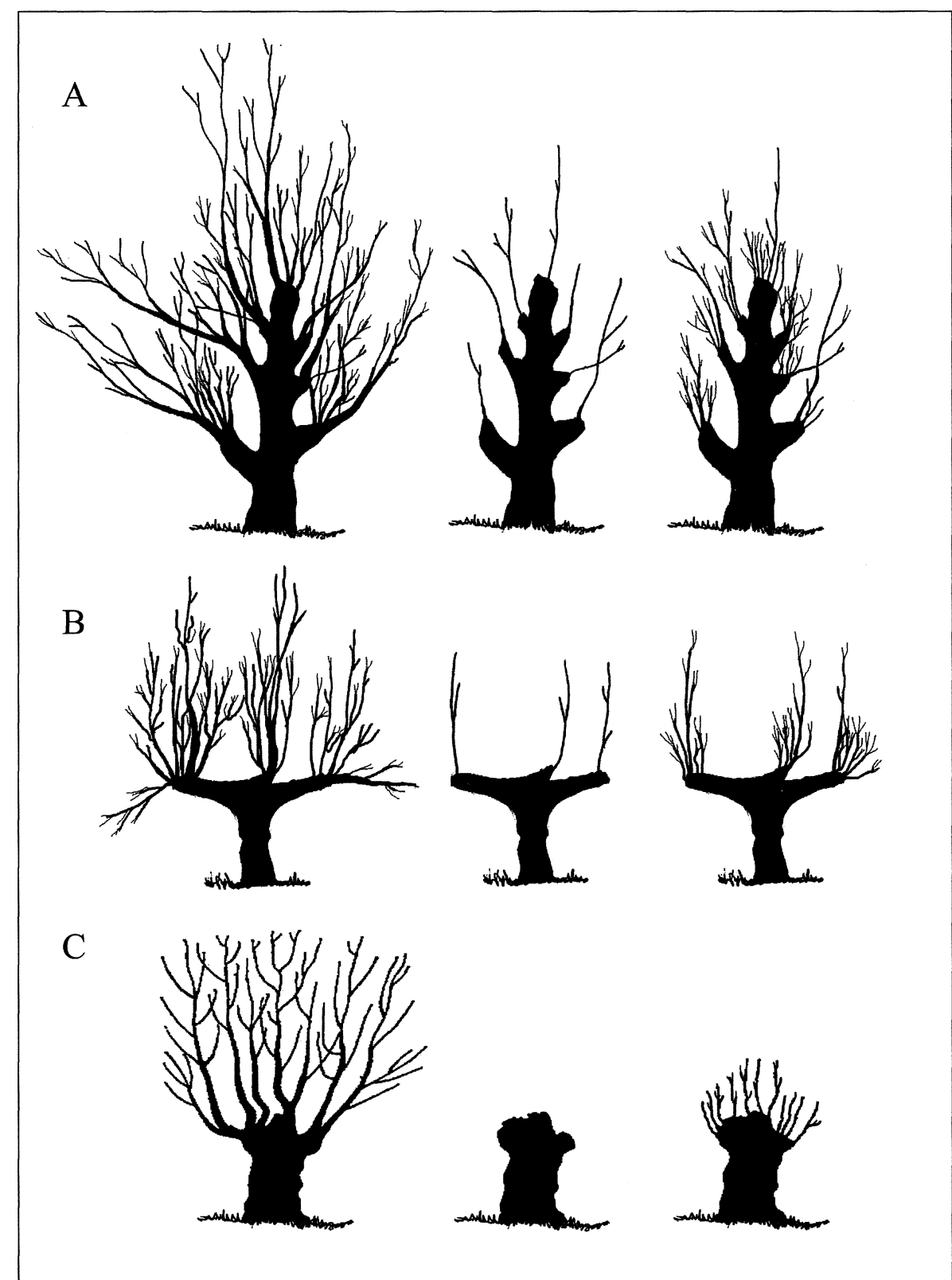

Fig. 5.-Formas adquiridas por los robles ( $A$ y $B$ ) y por el fresno (C)debido a las podas. Las figuras representan, de izquierda a derecha, al árbol el año antes de la poda, el año de la poda y el año después de la poda.

Estudios Geográficos, LXIV, 252, 2003 
Consecuencias de la transformación del bosque de la dehesa

La gestión del monte hueco.-La práctica de utilizar un mismo terreno para la cría de árboles y la manutención del ganado está extendida en todo el mundo, remontándose presumiblemente a periodos prehistóricos (Rackham, 1990). Ambos usos se encuentran completamente enfrentados ya que el ganado al pastar en el bosque actúa en contra de su regeneración. Contrariamente, el aumento de la densidad de árboles impide en gran medida el desarrollo del pasto del cual se nutre la ganadería. Como telón de fondo de este conflicto de intereses se encuentra la necesidad de la regeneración del arbolado. En el monte hueco existe la posibilidad de obtener distintos productos (bellotas, hojas verdes para el ganado, leña para el hogar y madera para manufacturas y construcción) sin que el terreno sea acotado al pastoreo. Sin embargo la regeneración tiene que producirse antes de que los árboles lleguen a su límite natural de edad, lo que implicaba la necesidad de acotar al pastoreo tarde o temprano. Esta necesidad se conoce desde antiguo y quedó especificada en las Ordenanzas de Buitrago, en algunas de cuyas ediciones se especificó que se debían acotar determinados montes por periodos de 10 años para su regeneración (Fernández, 1966; Flaquer, 1979).

Por las costumbres llegadas hasta el siglo xx sabemos que una de las formas de regenerar el arbolado era seleccionar de una mata de rebollos un vástago de buenas condiciones (rebollón). Esta regeneración de origen vegetativo es además más segura que la de origen sexual, ya que las plantas de semilla crecen en general más despacio lo que les hace más susceptibles al ganado, mientras que los rebrotes, especialmente los de algunas especies como el rebollo, crecen repetidamente a pesar de ser recomidos por el ganado ${ }^{13}$. Por tanto fue suficiente el acotamiento estacional, en primavera y verano, a cabras y ovejas para que proliferaran algunas matas de rebollo de las cuales se podría seleccionar posteriormente algún rebollón. A la larga el predominio de la regeneración vegetativa favorecería a Quercus pyrenaica, dada su gran capacidad de re-

${ }^{13}$ Aún así la regeneración vegetativa encuentra graves problemas cuando la presión ganadera es excesivamente alta. Así, en la descripción de algunos melojares de la provincia de Segovia en la segunda mitad del siglo XIX, se declaraba que estaban constituidos por una alfombra de brotes, incapaces de progresar debido a la intensidad de la presión ganadera (Laguna, 1864; Secall, 1889). 
brote vegetativo (Ruiz de la Torre, 1979). También es probable que la disminución coyuntural y no intencionada de la carga ganadera en determinadas épocas de la historia haya favorecido la regeneración. Esto justificaría la relativa homogeneidad en el tamaño de los árboles del primitivo monte hueco en general en toda la dehesa.

La creación del monte hueco modificó las condiciones del medio respecto al bosque original. La mayor insolación que llega al suelo se traduce en un agotamiento más rápido de las reservas hídricas. Al mismo tiempo la menor protección por las copas y la menor de sujeción del terreno por las raíces favorecen la erosión. A esto se suman los efectos indirectos del pastoreo (endurecimiento del suelo, pisoteo y removido del suelo, creación de sendas que pueden convertirse en regueros y hasta cárcavas tras lluvias torrenciales, etc.). En suma con el tiempo el sitio quedará empobrecido, con una pérdida de materia orgánica y elementos finos en el suelo y, en no pocos casos, con una perdida importante del suelo por erosión (Barnes et al., 1998). Las especies más tolerantes a la sequía, heliófilas y capaces de rebrotar tras ser comidas por el ganado, como es el caso del rebollo, incrementarán su superficie en detrimento de las especies más exigentes, como el roble templado o el arce.

Aunque la agricultura en los terrenos comunales y municipales estuvo penada severamente (Fernández, 1980), durante los siglos XIX y xx esta práctica se incrementó notablemente al dejar de tener vigor las Ordenanzas señoriales (Pardo and Gil, 1997). Sus efectos sobre el empobrecimiento del suelo en una zona de pendientes tan acentuadas, no requieren muchos comentarios. Una vez más será el rebollo la especie arbórea que mejor sorteará estos cambios regresivos sobre el medio debidos a la agricultura.

La heterogeneidad de la dehesa y su uso diferencial también ocasionarían que las consecuencias de la transformación del bosque fueran diferentes a lo largo de su superficie ${ }^{14}$. La división de la dehesa en huecos, orillas y medias orillas también ha sido importante en la transformación del bosque de la dehesa. En las lomas y laderas situadas en las medias orillas, zona periférica de la dehesa, la deforestación y pérdida de suelo ha sido mayor. En gran parte de estas zonas sólo ha quedado el

\footnotetext{
${ }^{14}$ En la actualidad parte de las zonas con suelos más empobrecidos están siendo ocupadas por encinas (Quercus rotundifolia), especie perennifolia menos exigentes que los robles, incluido Quercus pyrenaica.
}

Estudios Geográficos, LXIV, 252, 2003 
estrato de robles centenarios con ausencia de regenerado consecuencia de la baja producción de semilla, debido al decaimiento de los árboles, y también a las malas condiciones del medio para el regenerado (suelo esquelético o ausente y falta de cubierta arbórea). Estas zonas pueden ser calificadas de bosques fósiles (Quezel, 1977).

La forma de los árboles. - Las podas persiguen, al igual que el monte bajo, la obtención de productos de pequeño tamaño (principalmente leñas para consumo directo o para la fabricación de carbón). Existen dos tipos de poda empleados ampliamente a lo largo de la historia en toda Europa en zonas de aprovechamiento silvopastoral (Rackham, 1998): desmoche (corta de la guía principal a altura no superior a los 2-3 metros) y monda (corta de las ramas laterales). Con ambos tipos se buscaba alejar la zona de rebrote del diente del ganado (Grove and Rackham, 2001). La poda tradicional castellana de «horca $y$ pendón» es una forma muy próxima al desmoche, aunque cuando se dejaban varias horcas se llegaban a formas parecidas a las de los robles de la Puebla.

La norma de «horca y pendón», aprobada por los Reyes Católicos en 1496 (Mangas, 1981), fue modificada en 1574 por una instrucción que decía específicamente que no se dejara «ni horca ni pendón» sino que los árboles se «corten por lo alto desmochándolos a la redonda». Esta poda se acerca más a la monda que al desmoche, aunque se corta la parte final de la guía. Una nueva instrucción de 1748 obliga nuevamente a dejar «horca y pendón» pero se aclaraba que los árboles no se podían desmochar «por medio del tronco». Se trataba de evitar causar heridas graves que ponían en peligro la conservación de los árboles, debido a un tipo de poda que sólo algunas especies como el fresno soportan relativamente bien.

Algunas Ordenanzas de zonas cercanas daban más detalles sobre las podas. Por ejemplo en las del Sexmo de Lozoya (perteneciente a la Comunidad de Segovia) se especificaba que en cada árbol se debían de dejar tres aleros del grosor de una muñeca ${ }^{15}$. Esto se aproxima bastante a las podas tradicionales que se hacían en La Puebla, en las cuales se dejaban ramas con diámetros que rondaban los $10 \mathrm{~cm}$. En otras Ordenanzas, como las de la Villa de Riaza, se recalca el beneficio que supo-

15 Ordenanzas del Sexmo de Lozoya, en Saez Pombo (2000).

Estudios Geográficos, LXIV, 252, 2003 
nían las podas para la producción de bellota ${ }^{16}$. La bellota era muy importante en las economías locales tanto para la ganadería de autoconsumo (principalmente porcino) como para la que podía proporcionar algún excedente (sobre todo la lanar). El interés en la recogida de bellotas obligó a que se desarrollase su reglamentación (reparto proporcional entre vecinos, acotamiento de las zonas productoras, penas por entrar en las zonas acotadas, etc.). Otros lugares con presencia de robles de buenas dimensiones realizaban la recogida de bellota hasta mitad del siglo xx de manera parecida. Tal es el caso de Montejo de la Sierra y su monte el Chaparral (mas conocido como Hayedo de Montejo), con una población importante de Quercus petraea y Quercus pyrenaica de buenas dimensiones.

La forma de podar los robles en la dehesa de Puebla sigue el tipo tradicional castellano pero es, en general, menos agresiva con el árbol ya que en cada uno se dejan un número variable de «horcas y pendones» (normalmente más de tres). La longitud total del fuste es, en algunos casos, bastante más grande que en las especies sometidas a trasmocho, aunque no tan grande como las típicamente mondadas. Por esta razón permitía la obtención de maderas de dimensiones adecuadas para la construcción. La idea de respetar, en todos los pezones (u horcas), un renovizo (o pendón) en cada turno servía para facilitar la brotación en el siguiente periodo vegetativo, al actuar esta rama de «tirasavias». Este tipo de poda debió de estar bastante extendido en la comarca. Puede ser observado tanto en otras dehesas boyales como dispersos en zonas poco accesibles de la sierra. No solo en las especies citadas sino también en quejigos (Quercus fagínea), hacia el sureste, e incluso en abedules (Betula pendula) en la dehesa de La Hiruela (Madrid) y hayas (Fagus sylvatica) en Montejo de la Sierra (Madrid) y Peñalba de la Sierra (Guadalajara). En todos estos casos se ha sobrepasado el turno de poda y los brotes poseen dimensiones que suelen superar los 10 centímetros.

En relación con el tamaño de los árboles, las Ordenanzas de Buitrago no especificaban la equivalencia entre el «marco» (tamaño máximo de

\footnotetext{
${ }^{16}$ Uno de los artículos de estas Ordenanzas decía lo siguiente: «... para que mejor puedan crecer los arboles y hagan cabeza de fruto... ... los dichos montes tienen necesidad de entresacarse y podarse las ramas... ... que se pode e limpie, cortando los pies retuertos. $Y$ en las partes donde estuviere muy espeso el monte se entresaque e pode para lo que quedare pueda criar y hacer cabeza de fruto».
}

Estudios Geográficos, LXIV, 252, 2003

$$
-426-
$$


los árboles que podían ser cortados) y el diámetro de los árboles a los que correspondía. Este tamaño, sin duda, debía tener relación, o bien con la existencia de árboles de porte suficiente para producir bellota, lo que incrementaría su valor en una economía de fuerte base ganadera, o con la posibilidad de obtener trozas de madera de dimensiones suficientes para ser empleadas en la construcción, hecho que sin duda incrementaría su valor. Puede que el marco se refiriera a árboles que tenían una circunferencia de una vara castellana $(0,836 \mathrm{~m})$ o lo que es lo mismo un diámetro de $26,6 \mathrm{~cm}$, cifra que se aproxima a los $30 \mathrm{~cm}$ de diámetro a partir de los cuales comenzaban a ser podados los robles en la Puebla. Por otro lado mientras las podas se hacían en el momento que llegaba el turno a cada una de las áreas en las que se dividía el monte, la corta de árboles de grandes dimensiones requería el permiso del ayuntamiento. En cualquier caso queda clara la importancia de las dimensiones de los árboles en la configuración del bosque de la dehesa así como el tipo de aprovechamiento predominante basado en la realización de podas que favoreció la presencia de un estrato de árboles viejos.

\section{Características actuales de la dehesa}

En la actualidad existe una porción importante de la dehesa con una marcada degradación por pérdida de suelo ${ }^{17}$. En las vaguadas y barrancos (los huecos), zonas donde mejor se ha conservado el suelo, predominan los robles y fresnos podados de avanzada edad con densidades qué, en ocasiones, alcanzan los 200 pies por hectárea. Entre estos grandes árboles, que superan los $50 \mathrm{~cm}$ de diámetro, aparecen mezclados otros de las mismas especies pero de mucha menor edad, con una cubierta bastante completa que alcanza los 4000 pies por hectárea, y sotobosque de brezo blanco (Erica arborea). En estas zonas de vaguada es frecuente además observar otras especies arbóreas acompañantes como el arce (Acer monspesulanum), el mostajo (Sorbus aria) o el serbal de cazadores (Sorbus aucuparia). Las manchas de monte denso sin árboles trasmochados, tratadas como monte bajo para leñas, son escasas. En el resto de la dehesa la vegetación predominante es el matorral,

${ }^{17}$ Según la cartografía de la Comunidad de Madrid, a escala 1:50.000, las zonas arboladas constituyen el 39,7 por ciento de la superficie de la dehesa.

Estudios Geográficos, LXIV, 252, 2003 
principalmente de brezo blanco y jara (Cistus ladanifer), con o sin arbolado disperso, cuya densidad no sobrepasa en general los 40 pies por hectárea. Estas cifras son similares a las observadas en el monte hueco de otras dehesas de la zona como El Hayedo de Montejo o las dehesas boyales de Peñalba de la Sierra y la Hiruela. La vegetación de los arroyos de la Puebla y del Portillo está constituida por un bosque de galería con alisos (Alnus glutinosa), álamos temblones (Populus tremula), sauces (Salix sp) y fresnos (Fraxinus angustifolia), con trasmochos de roble y estrato arbustivo predominante de brezo. Aparecen esparcidos por el monte pastizales naturales, muy mermados en su superficie por la expansión de la jara (apenas cubren el 4 por ciento).

Con la disminución de la población, y su envejecimiento, y la aparición de nuevas fuentes energéticas se han ido abandonando poco a poco los aprovechamientos tradicionales, sobre todo en las zonas más alejadas de la dehesa. A esto ha contribuido la mayor dificultad que supone la realización de podas frente a la corta del monte bajo y la escasez, cada vez mayor, de caballerías para realizar el transporte de la leña hasta el pueblo. El resultado del abandono de las podas es que las ramas, provenientes de brotes adventicios situados en los pezones, crecen excesivamente corriendo el riesgo de desgajarse por el viento, la nieve o el peso propio. Por otro lado, a partir de las heridas realizadas con las podas se producen pudriciones, que de no eliminar la rama afectada se introducen progresivamente hasta llegar al tronco. Poco a poco se ha ido produciendo un empeoramiento del estado fitosanitario de los árboles. Cuando estos están muy debilitados el embate del viento puede derribarlos. Actualmente la mayor parte del arbolado de mayor edad se encuentra en una situación inestable y en muchas zonas existe un alto porcentaje de árboles muertos recientemente. Son muy pocos los rodales que aún se encuentran en turno, es decir que fueron podados hace menos del turno de corta, cuyos árboles aún poseen cargas estabilizadas. Se localizan en las zonas más accesibles.

\section{Conclusiones}

$\mathrm{El}$ aprovechamiento realizado en la dehesa durante el siglo xx, tiene la permanencia de lo tradicional en el mundo agrario, sin sufrir escasas modificaciones desde la Edad Media. Se trataría de un uso intensivo

Estudios Geográficos, LXIV, 252, 2003

$$
-428-
$$


pero, a la vez, conservador de los recursos naturales necesarios para la economía tradicional, como ha sido denominado por Rackham (1990), en referencia al uso medieval de los bosques medievales ingleses. La transformación del bosque consistió en la creación de un agroecosistema en el que el hombre participaba activamente pero no eliminó por completo las características de naturalidad del bosque anterior. En este fenómeno de coevolución, las sociedades humanas y las comunidades naturales se han transformado mutuamente a lo largo del tiempo llegando, en muchos casos, a encontrar un equilibrio o relativa estabilidad a lo largo del tiempo.

Dentro de un espacio natural de este tipo biodiversidad y uso tradicional están altamente relacionados, condicionando ambos las políticas de gestión (Peterken, 1993). En primer lugar se debe procurar el mantenimiento de los distintos tipos de hábitats, la conservación de todas las especies, aunque no necesariamente en sus proporciones actuales (favoreciendo la regeneración natural siempre que sea posible), y la consecución, a largo plazo, de una estructura balanceada de edades donde tengan cabida tanto los árboles maduros como el regenerado (Fuller and Peterken, 1995). Estos autores señalan también que en los bosques sometidos tradicionalmente a pastoreo se debe favorecer la supervivencia de los árboles mayores (centenarios) y los hábitats asociados a ellos, recuperando si es preciso las podas tradicionales, y el mantenimiento de la estructura en mosaico conseguida mediante la aplicación de distintas intensidades de pastoreo. Otra de las prioridades en la gestión de estos espacios se relaciona con la necesidad de regeneración y de solventar las pérdidas de suelo. Pese a los efectos positivos que a largo plazo ha tenido la gestión tradicional de la dehesa también ha conllevado otros procesos negativos. En la parte central de la dehesa las masas poseen un alto grado de naturalidad. Sin embargo conforme ascendemos ladera arriba, hacia las orillas y medias orillas, la degradación ha sido mayor. La recuperación de gran parte de estas zonas es difícil debido a la presencia de suelos raquíticos.

El relativo buen grado de conservación de la parte central de la dehesa, con árboles de gran porte y una estructura tendente a la irregularidad, haría posible una gestión encaminada a la conservación estricta de ciertas porciones de esta zona. Los bosques poco alterados tienen en la actualidad un gran interés dentro del contexto europeo, dada la gran reducción y transformación sufrida por los espacios forestales a lo lar-

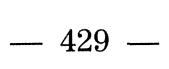

Estudios Geográficos, LXIV, 252, 2003 
go de la historia. Se considera necesaria su conservación y protección para que puedan servir de ejemplos vivos de uno de los hábitats menos abundantes del continente europeo y como controles de los cambios que puedan experimentar otros ecosistemas (Peterken, 1996). Dentro de la dehesa «los huecos» poseen algunas características propias de los bosques poco alterados como es la presencia local de árboles viejos y madera muerta y sus hábitats asociados. La conservación de ciertas porciones de la dehesa debe enmarcarse dentro de una política realista y coherente que no tenga su contrapartida en la explotación y degradación excesiva de otras áreas próximas. En el caso de la dehesa de la Puebla la conservación de los huecos no debe significar la explotación elevada de otras áreas, ya de por sí bastante degradadas, como las partes periféricas del monte.

La conservación de los habitats de interés de la dehesa precisa en la actualidad del apoyo científico y técnico. En primer lugar para identificar los aspectos que más enriquecen sus ecosistemas y en segundo lugar para proponer actuaciones técnicamente posibles y, socio-económicamente viables. La gestión de este espacio debería partir de investigaciones que determinen las producciones y potencialidades de producción de leñas, posibilidades de su transformación, cargas ganaderas, tipo y/o combinaciones de ganado que mejoren y mantengan la calidad de los pastos y del suelo, técnicas selvícolas para la regeneración y aprovechamientos, etc. En definitiva el estudio de los factores que garanticen una sostenibilidad ambiental y social.

En las dos últimas décadas las organizaciones conservacionistas (estatales y no gubernamentales) han hecho hincapié en la necesidad de integrar conservación y desarrollo, siendo un elemento fundamental para el éxito de los proyectos de conservación la generación de beneficios económicos para la población local (Shuterland, 2000). Con ello se buscaría en cierta medida la reapropiación de los recursos, favoreciendo su perdurabilidad. En el caso de la dehesa de La Puebla, la conservación se verá facilitada si se armonizan adecuadamente los distintos usos, principalmente la ganadería, la obtención de leñas y el turismo rural. Uno de los mayores valores que posee un espacio como la dehesa de la Puebla es su herencia cultural, desaparecida en un buen número de dehesas de la zona transformadas casi en su totalidad en montes bajos de rebollo y con escasez de árboles viejos. En este sentido la demanda social de espacios naturales y rurales constituye una oportunidad para

Estudios Geográficos, LXIV, 252, 2003 
desarrollar programas de educación ambiental en la dehesa relacionados con su riqueza ecológica y etnográfica. Dentro de este último aspecto será fundamental la conexión entre las distintas administraciones, desde la municipal a la estatal, para conseguir el tipo de inversiones y apoyos necesarios para poner en práctica estos programas.

De lo dicho a lo largo de este trabajo se deduce que la gestión de un espacio natural como el aquí estudiado debe integrar la conservación y el uso tradicional. Por esta razón el estudio de los aprovechamientos tradicionales y de su adaptación a las condiciones locales, como base para construir nuevos modelos productivos sostenibles que integren modernidad y conocimiento tradicional, cobra gran importancia en la conservación de ecosistemas a la vez altamente humanizados pero de gran valor biológico.

\section{Agradecimientos}

A los vecinos de Puebla de la Sierra, especialmente a sus mayores, por ofrecernos cuanta información les solicitamos para poder reconstruir el aprovechamiento tradicional de su dehesa boyal.

\section{BIBLIOGRAFÍA}

Barnes, B. V.; ZaK, D. R.; Denton, S. R. and SpurR, S. H. (1998): Forest ecology. Jon Wiley \& Sons, New York, 774 pp.

FERNÁNDEZ, M. (1966): Fuentes para la historia de Buitrago y su tierra. Volumen primero. Edición del autor, Madrid, $198 \mathrm{pp}$.

(1980): Buitrago y su tierra (algunas notas históricas). Edición del autor, Madrid, $324 \mathrm{pp}$.

FLAQUER, R. (1979): «El aprovechamiento de los comunales: las Ordenanzas de Buitrago». Agricultura y Sociedad, 2: 323-370.

Fuller, R. J. y Peterken, G. F. (1995): «Woodland and scrub». En: W. J. Sutherland and D. A. Hill (Editors), Managing habitats for conservation. Springer-Verlag, New York., pp. 327-361.

GARCía DE CORTAZAR, J. A. (1990): La sociedad rural en la España medieval. Siglo XXI, Madrid, 320 pp.

Grove, O. T. y RACKHAM, O. (2001): The nature of Mediterranean Europe. An ecological history. Yale University Press, London.

GRupo73 (1973): La economía del Antiguo Régimen. El Señorío de Buitrago. Universidad Autónoma, Madrid, $230 \mathrm{pp}$.

LAGUNA, M. (1864): Memoria de reconocimiento de la Sierra de Guadarrama bajo el punto de vista de la repoblación de sus montes. Imprenta Nacional, Madrid, $47 \mathrm{pp}$.

Estudios Geográficos, LXIV, 252, 2003

$$
-431-
$$


MANGAS, J. M. (1981): El régimen comunal agrario de los concejos de Castilla. Ministerio de Agricultura. Secretaría General Técnica, Madrid, $316 \mathrm{pp}$.

MarTín, E. (2001): Primera revisión de la Ordenación del Monte de U. P., n. ${ }^{\circ} 109$ «Dehesa boyal». Término Municipal de Puebla de la Sierra. Proyecto Fin de Carrera. E.T.S. Ingenieros de Montes, Madrid., $135 \mathrm{pp}$.

MEIGGS, R. (1982). Trees and timber in the ancient Mediterranean world. Clarendon, Oxford, $555 \mathrm{pp}$

Montero, G.; SAN Miguel, A. y CAÑellas, I. (1998): «Systems of Mediterranean agriculture: "La Dehesa"». En: R. Jiménez and J. Lamo de Espinosa (Editors), Sustainable agriculture. Agrofuturo, LIFE and Mundiprensa,, Madrid, pp. 519-554.

PARDO, F. y GIL, L. (1997): «La transformación del paisaje de la Sierra Pobre de Madrid. Influencia de la agricultura y la ganadería en la extinción local de los pinares». Estudios Geográficos, LVII (228): 397-423.

Peterken, G. F. (1993): Woodland conservation and management. Chapman \& Hall, London, $374 \mathrm{pp}$.

Peterken, G. F. (1996): Natural woodland. Ecology and conservation in northern temperate regions. Cambridge University Press, New York, $522 \mathrm{pp}$.

QuEZEL, P. (1977): «Mediterranean forests». En: Mediterranean forest and maquis: ecology, conservation and management. UNESCO, Paris, pp. 1-32.

RACKHAM, O. (1990): Trees and woodland in the British landscape. Weidenfeld \& Nicolson, London, $234 \mathrm{pp}$.

RACKHAM, O. (1998): «Savannah in Europe». In: K. J. Kirby and C. Watkins (Editors), The ecological history of European forests. CAB International, New York, pp. 1-24.

RUIZ DE LA TORRE, J. (1979): Árboles y arbustos de la España peninsular. Escuela Técnica Superior de Ingenieros de Montes, Madrid, $512 \mathrm{pp}$.

SÁEz, E. (1953): Los Fueros de Sepúlveda. Diputación Provincial de Segovia, Segovia, 921 pp.

SAEz Pombo, E. (2000): Montes públicos, territorio y evolución del paisaje en la Sierra Norte de Madrid. Comunidad de Madrid-Universidad Autónoma de Madrid, Madrid, $245 \mathrm{pp}$.

ScotT, S. P. (1910): The Visigothic Code (Forum judicum). Boston Book Company, Boston, $409 \mathrm{pp}$.

SECALL, J. (1889): «Apuntes, noticias y datos de una excursión forestal». Revista de montes, 292: 143-148.

SHUTERLAND, W. J. (2000): The conservation handbook. Research, management and policy. Blackwell Science, Oxford, $271 \mathrm{pp}$.

TORquemada, M. J. (1997): La protección ecológica de la Castilla bajomedieval. Servicio de Publicaciones de la Facultad de Derecho. Universidad Complutense de Madrid., Madrid, $156 \mathrm{pp}$.

Ubieto, A. (1959). Colección diplomática de Riaza (1258-1457). Diputación Provincial de Segovia, Segovia, $241 \mathrm{pp}$.

RESUMEN: Se ha estudiado la gestión tradicional de una dehesa boyal localizada en un área montañosa del centro de España, en el municipio madrileño de Puebla de la Sierra, y sus efectos sobres las características del bosque. La dehesa, con 336 hectáreas, tiene una posición estratégica dentro del término municipal, por su proximidad al casco urbano y su localización intermedia respecto a los terrenos comunales más alejados. Está compuesta principalmente de robles melojos (Quercus pyrenaica), con la presencia puntual de otras especies de gran interés geobotánico como el roble albar (Quercus petraea).

Los precedentes más antiguos de la gestión de la dehesa tienen su origen en la Edad Media, época en la que se sientan las bases y generalizan dos formas de aprovecha-

Estudios Geográficos, LXIV, 252, 2003 
miento del bosque que perdurarán hasta la actualidad: el monte bajo y el monte hueco. La dehesa de Puebla fue utilizada fundamentalmente en régimen de monte hueco llegando las tradiciones de uso hasta el siglo xx. Las informaciones de hombres y mujeres de edad superior a los 70 años ha permitido reconstruir esta gestión tradicional.

En el trabajo se analiza el aprovechamiento estacional de la dehesa por los distintos tipos de ganado (vacuno, lanar y cabrío). Se describe la forma de podar los árboles y las reglas que regulaban las podas para las distintas especies arbóreas. También se discuten los efectos que la gestión tradicional ha tenido sobre las características del bosque, tanto en estructura como en composición. Finalmente se analiza el impacto del uso tradicional del bosque en la conservación de este espacio. Se destaca la importancia de un modelo de gestión que mantiene la estructura original y permite la permanencia de un buen número de características de naturalidad (presencia de grandes árboles, diversidad, heterogeneidad, etc.). Además se destaca la necesidad actual de integrar las formas de aprovechamiento tradicional (protagonizado por la población local), el apoyo técnicocientífico (para mejorar las técnicas de conservación de este espacio) y el uso culturaleducativo como pilares básicos de la gestión de este espacio natural de gran interés biológico y cultural.

PALABRAS ClAVE: gestión tradicional, ganado, bosque, poda.

ABSTRACT: The traditional management of an open wood for the feeding of ploughing cattle (dehesa boyal), located in a mountain area of Central Spain, was studied. The study area is situated in Puebla de la Sierra (Madrid). The dehesa has 336 ha and is strategically situated between the communal lands of the higher slopes and the village. The main tree species is melojo oak (Quercus pyrenaica) with other less frequent species interesting from a conservation point of view like sessile oak (Quercus petraea).

The management rules in the dehesa originated in medieval times, with two main types of wood management: the coppice and the open wood pasture (monte hueco). The dehesa of La Puebla was mostly used as an open wood pasture until the $20^{\text {th }}$ century. Characteristics of the traditional management of the dehesa have been recreated through interviews to the old inhabitants of the village.

The seasonal grazing by cows, sheep and goats is described for different parts of the dehesa. Lopping for the different tree species and the rules that regulated them are described. Also are discussed the effects of traditional management on the structure and composition of the dehesa wood. Finally, the importance of the woodland traditional management for nature conservation is analysed. The utility of the management rules studied, which does not change a large quantity of the forest characteristics (large trees, diversity, heterogeneity, etc.), is pointed out. Also is highlighted to integrate the traditional management for local people, scientific support for the improvement of the nature conservation methods and the cultural and educational programs in the planning of this highly interesting nature reserve.

KEY wORDS: traditional management, livestock, woodland, lopping.

RÉSUMÉ: On a étudié la gestion traditionnelle du pâturage appelé « déhesa boyal », situé dans une zone montagneuse du centre Espagne dans le municipe madrilène de Puebla de la Sierra ainsi que ses effets sur les caractéristiques du bois. Le pâturage comprend 336 hectares et a une position stratégique dans le domaine local quant à sa proximité à l'enceinte de la ville et à sa localisation intermédiaire par rapport aux terrains communales plus éloignés. Il est principalement composé de chênes tauzins (Quercus pyrenaica), avec 
la présence ponctuelle d'autres espèces de grand intérêt naturel tel que la chêne sessile (Quercus petraea).

Les antécédents les plus anciens de la gestion du pâturage ont leurs origines dans le Moyen Âge, une époque où ses bases sont constituées et où deviennent générales deux formes de profit du bois qui perdureront jusqu'aux nos jours : le taillis et le pâturage entouré d'arbres ( «monte hueco »). Le pâturage de Puebla a été fondamentalement utilisé comme un «monte hueco », les traditions de cet usage durant jusqu'au le Xxème siècle. Les informations sur des hommes et des femmes d'un âge supérieur aux 70 ans ont permis la reconstruction de cette gestion traditionnelle.

Dans ce travail on décrira le profit saisonnier de différents parties du pâturage selon les types de bétail (principalement le bovin, le caprin et celui à laine). On décrira la manière d'émonder les arbres et le règlement établit par l'émondage de chaque espèce. On discutera aussi à propos des effets de la gestion traditionnelle sur les caractéristiques du bois en ce qui concerne sa structure et sa composition. Enfin, on analysera l'utilité de l'usage traditionnel du bois pour la conservation de cet espace. On focalisera sur l'importance de ce modèle de gestion, n'étant excessivement transformateur de la nature originaire, pour la conservation d'un grand nombre de caractéristiques de naturalité (présence de grands arbres, diversité, hétérogénéité, etc.) D'ailleurs, on remarque la nécessité actuelle d'intégrer les formes de profit traditionnel (représenté par la population actuelle), l'appui scientifique ( pour améliorer les techniques de conservation de cet espace) et l'usage culturel et éducatif comme des piliers basiques de la gestion de cet espace naturel de grand intérêt biologique et culturel.

Mots CLES: gestion traditionnelle, bétail, bois, émondage. 